\title{
Asleep Surgery May Improve the Therapeutic Window for Deep Brain Stimulation of the Subthalamic Nucleus
}

\author{
Farhad Senemmar, DMD* ๑; Christian J. Hartmann, MD*; \\ Philipp J. Slotty, MD $^{\dagger}$; Jan Vesper, MD $^{\dagger}{ }^{\circ}$; Alfons Schnitzler, MD*; \\ Stefan Jun Groiss, MD* $\odot$
}

\begin{abstract}
Objective: The effect of anesthesia type in terms of asleep vs. awake deep brain stimulation (DBS) surgery on therapeutic window (TW) has not been investigated so far. The objective of the study was to investigate whether asleep DBS surgery of the subthalamic nucleus (STN) improves TW for both directional (dDBS) and omnidirectional (oDBS) stimulation in a large single-center population.

Materials and Methods: A total of 104 consecutive patients with Parkinson's disease (PD) undergoing STN-DBS surgery (80 asleep and 24 awake) were compared regarding TW, therapeutic threshold, side effect threshold, improvement of Unified PD Rating Scale motor score (UPDRS-III) and degree of levodopa equivalent daily dose (LEDD) reduction.

Results: Asleep DBS surgery led to significantly wider TW compared to awake surgery for both dDBS and oDBS. However, dDBS further increased TW compared to oDBS in the asleep group only and not in the awake group. Clinical efficacy in terms of UPDRS-III improvement and LEDD reduction did not differ between groups.

Conclusions: Our study provides first evidence for improvement of therapeutic window by asleep surgery compared to awake surgery, which can be strengthened further by dDBS. These results support the notion of preferring asleep over awake surgery but needs to be confirmed by prospective trials.
\end{abstract}

Keywords: Anesthesia, asleep, DBS, PD, STN, therapeutic window

Conflict of Interest: Farhad Senemmar reports no conflict of interest. Christian J Hartmann received honoraria and travel expenses from Abbott Medical. Philipp J. Slotty received speaker honoraria and travel reimbursement from Abbott Medical. Jan Vesper received travel expenses from Medtronic, Abbott and Boston Scientific, consultancies of Abbott, Boston Scientific, Medtronic, Biotronik, Sparks Therapeutic, UniQure, grants from German Research Council, Medtronic. Alfons Schnitzler reports grants from German Research Council; personal fees from Medtronic Inc, Boston Scientific, Abbott Medical, UCB and Teva Pharma. Stefan Jun Groiss received honoraria and/or travel expenses from Abbott Medical, Actelion, Boston Scientific, Medtronic, Rogue Research and UCB.

\section{INTRODUCTION}

Parkinson's disease (PD) is one of the most widespread neurodegenerative disorders and is characterized by its cardinal motor symptoms bradykinesia, rigidity, and tremor, which are caused by the loss of dopaminergic neurons in the substantia nigra (SN) pars compacta, and additionally by mental disorders like depression and psychosis $(1,2)$.

Although levodopa is a highly effective medical treatment of motor symptoms in PD, long-term treatment lead to adverse effects like dyskinesia and motor fluctuations and is often not satisfactory $(3,4)$.

Address correspondence to: Stefan Jun Groiss, MD, Department of Neurology, Heinrich-Heine University Düsseldorf, Moorenstr. 5, 40225 Düsseldorf, Germany. Email: groiss@uni-duesseldorf.de

* Department of Neurology \& Institute of Clinical Neuroscience and Medical Psychology, Medical Faculty, Heinrich-Heine University Düsseldorf, Düsseldorf, Germany; and

+ Department of Functional Neurosurgery and Stereotaxy, Neurosurgical Clinic, Medical Faculty, Heinrich-Heine University Düsseldorf, Düsseldorf, Germany

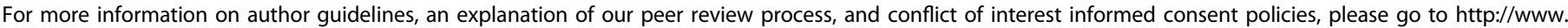
wiley.com/WileyCDA/Section/id-301854.html

Source(s) of financial support: The authors received no specific funding for this work. The work has not been presented previously

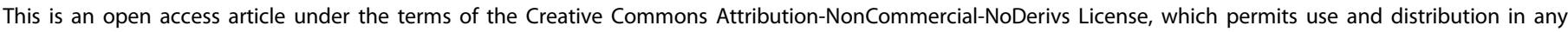
medium, provided the original work is properly cited, the use is non-commercial and no modifications or adaptations are made.

www.neuromodulationjournal.com 
Deep Brain Stimulation (DBS) has been shown to be an effective treatment for patients with advanced PD suffering from motor complications refractory to medication $(3,5)$.

Recently, a new generation of electrodes with segmented contacts was introduced, which allow the stimulation to be delivered directionally in an axially asymmetric way (6-8). With this new lead design, stimulation is supposed to be delivered more precisely with less effect on adjacent areas (9). Several studies could show that directional deep brain stimulation (dDBS) results in a wider therapeutic window (TW), defined by the stimulation current difference between therapeutic threshold (TT) and side effect threshold (SET), for both subthalamic nucleus (STN)- and VIM-DBS $(6,8,10,11)$.

Regarding anesthesia, DBS can be performed with the patient awake, which allows the operating team to communicate with the patient and to test the stimulation effects intraoperatively, or asleep, which was commonly used for patients who were not suitable for awake surgery due to different reasons like unbearable motor or nonmotor off-medication symptoms, anxiety, cardiovascular problems or other medical limitations $(12,13)$. It is worth mentioning that definition of both asleep and awake surgery may vary throughout the literature. Nowadays, asleep surgery is often used for purely imaging-based targeting in general anesthesia and without microelectrode recording (MER) (14). However, modified anesthesia protocols have been introduced recently, where sedation was reduced during general anesthesia surgery thus allowing for sufficient MER and some clinical testing with the patient still asleep (15).

Direct comparisons of asleep and awake DBS surgery using the modified anesthesia protocol and MER with adequately matched groups are scarce $(16,17)$. The first study reported superiority of awake surgery in a small sample study more than ten years ago (17). In a more recent study with much larger sample size, we could show that awake surgery may be still advantageous in regard of the speed of motor function improvement, which was faster in the awake group. However, the difference between both groups ceased already after one year thus questioning the clinical relevance.

Recent meta analyses found comparable clinical outcomes of asleep DBS to those of awake surgery (18-20). Ho et al. retrospectively compared 139 patient cohorts awake with 16 patient cohorts (seven cohorts with MER) asleep and found no significant differences in motor outcomes and levodopa equivalent daily dose (LEDD) (20). Wang et al. analyzed 59 articles published through 19 years and also found comparable results between asleep and awake surgery in terms of clinical as well as surgical outcomes (18). This is generally in line with the most recent study of Liu et al. who systematically reviewed the literature involving 14 cohort studies and found no significant differences in motorc outcomes and LEDD (19). However, as already mentioned asleep and awake surgery may have been defined differently among the included studies.

Although increasing numbers of literature comparing asleep vs. awake DBS surgery are available, most of them compared motor outcome in terms of Unified Parkinson Disease Rating Scale (UPDRS) motor score. The effect of anesthesia type on therapeutic window, which has recently been found a good measure for DBS outcome, has not been investigated so far $(16,21,22)$. Moreover, all studies were performed on patients with conventional ring electrodes. Here, we therefore investigated for the first time how asleep DBS surgery influences the therapeutic window and whether its effect can be strengthened with directional stimulation.

\section{MATERIALS AND METHODS}

The study was approved by the local ethics committee of the University Düsseldorf and was conducted in accordance to the Declaration of Helsinki.

\section{Patients}

A total of 104 consecutive PD patients (75 male, 29 female) who underwent bilateral STN-DBS surgery using directional leads at the center of movement disorders in Düsseldorf from 2016 to 2019 were retrospectively analyzed. A total of 80 received electrode implantation asleep (56 male, 24 female), while 24 PD patients were partially awake during surgery ( 19 male, 5 female). Four patients did not require bilateral stimulation at the beginning and were therefore stimulated unilaterally at the time of evaluation. Baseline characteristics are shown in Table 1.

\section{Neurosurgical Procedure}

The surgical procedure has been described in detail elsewhere (16). In brief, both awake and asleep surgeries were conducted off dopaminergic medication for at least 12 hours before surgery, except for subcutaneous apomorphine, which was discontinued in the morning of surgery $(12,23)$. After sedation with propofol and remifentanil, the patient's head was fixed on a stereotactic Leksell frame. Stereotactic planning was performed on preoperative MRI fused with intraoperative CT. Stereotactic coordinates based on anatomical structures $(12 \mathrm{~mm}$ lateral from midline, $2.3 \mathrm{~mm}$ behind midcommissural point and $3 \mathrm{~mm}$ below AC-PC line) were used to define the target point. After skin incision and drilling of the burr hole sedation was completely stopped in the awake group and reduced up to $200 \mathrm{mg} / \mathrm{h}$ for propofol and $0.4 \mathrm{mg} / \mathrm{h}$ for remifentanil, if possible, in the asleep group. A BenGun system with up to five parallel trajectories with $2 \mathrm{~mm}$ distance among each other, were used for MERs. Simultaneous MER of all the parallel trajectories were conducted in $1 \mathrm{~mm}$ steps starting $10 \mathrm{~mm}$ above the calculated target point, which was supposed to be at the dorsal border of the STN and continued until

Table 1 Comparison of Baseline Characteristics.

$\begin{array}{llll}\text { Baseline characteristics } & \text { Asleep } & \text { Awake } & p \text { value } \\ \text { Age (years) } & 63.03 & 58.39 & <0.05 \\ \text { Disease duration (years) } & 9.2 & 9.3 & \mathrm{Ns} \\ \text { H \& Y stage } & 2.58 & 2.53 & \mathrm{Ns} \\ \text { UPDRS off medication pre } & 33.21 & 33.10 & \mathrm{Ns} \\ \text { LEDD pre (mg) } & 945 & 1027 & \mathrm{Ns} \\ \text { MDRS pre } & 140.0 & 140.8 & \mathrm{Ns} \\ \text { MoCA pre } & 24.80 & 25.75 & \mathrm{Ns} \\ \text { Number of MER trajectories } & 3.36 & 3.74 & <0.05 \\ \text { Duration of surgery (cut - seam, min) } & 175.4 & 181.7 & \mathrm{Ns}\end{array}$

MDRS, Mattis Dementia Rating Scale; MoCA, Montreal Cognitive Assessment; UPDRS, Unified Parkinson Disease Rating Scale; LEDD, levodopa equivalent daily dosage; H\&Y, Hoehn and Yahr; MER, micro-electroder Recording. 
the lower border of the STN was identified to allow for a complete mapping of the STN. The number of inserted trajectories was not based on the type of anesthesia in terms of awake or asleep surgery but was decided on the basis of the patient's individual anatomy and risk of hemorrhage. Then, intraoperative test stimulation was followed to define $\Pi$ T and SETs (13). First, in both groups, stimulation parameters were set to low frequency stimulation at $4 \mathrm{~Hz}$ with $210 \mu \mathrm{sec}$ pulse width and $6 \mathrm{~mA}$ intensity in order to provoke and recognize muscle twitching by pyramidal tract stimulation and then switched to high-frequency stimulation at $130 \mathrm{Hy}, 60 \mu \mathrm{sec}$ pulse width, where stimulation intensity was increased stepwise until sustained side effects could be observed or a maximum of $5 \mathrm{~mA}$. While direct communication with the patient and thus testing of both effects and different side effects was possible in the awake group only, testing in the asleep group was limited to muscle contractions due to pyramidal tract stimulation. After defining the final target for lead placement, segmented DBS electrodes provided by either Boston Scientific (16 patients) or Abbott/St. Jude Medical (88 patients) were implanted. Lead placement was monitored online by intraoperative X-ray and confirmed by CT-scan postoperatively. The pulse generator was implanted either on the same day or within few days after externalization and test stimulation.

\section{Outcome Values}

Therapeutic Windows

Within the first three month after surgery, a monopolar review of all contacts was carried out in order to determine the $\Pi$, defined as the lowest stimulation amplitude leading to clinically relevant improvement of motor function, and the SET, defined as the lowest stimulation amplitude leading to persistent side effects. The primary outcome value, the TW was calculated as difference between TT and SET. TW was calculated in absolute stimulation amplitude (mA) and relative to $\Pi$ (TW\%). The segment with the widest TW was defined as best directional DBS (dDBS) and compared to omnidirectional DBS (oDBS) for both asleep and awake groups.

\section{Motor Improvement}

Improvement of motor function was measured by the degree of reduction of UPDRS III score immediately after activation of stimulation after three months and one year postoperatively (change from Med OFF/Stim OFF to Med OFF/Stim ON) and compared between both groups.

\section{Medication}

Degree of LEDD reduction after three months and one year postoperatively were compared between the two groups $(24,25)$.

\section{Chronic Stimulation Amplitude}

Chronic stimulation amplitudes after three months and one year postoperatively were compared between the asleep and awake group.

\section{Baseline Values}

We also compared age, disease duration, Hoehn and Yahr stage, preoperative UPDRS III off medication, preoperative LEDD, cognition measured by preoperative Dementia Rating Scale (MDRS) and Montreal Cognitive Assessment (MoCA), number of microelectrode trajectories, duration of surgery (time frame between first cut and first seam) between the groups.

\section{Statistics}

For statistical analysis, we used GraphPad Prism 5 for Windows. All data are presented by means with standard error (SE). Shapiro-Wilk test was used to test for normality and Mann-Whitney$\mathrm{U}$-Test was used for further analysis. Significance level was set to $p<0.05$ except for analysis of TW and TW\%, TT and SET, where significance level was adapted to $p<0.025$ to correct for multiple comparisons.

\section{RESULTS}

\section{Baseline Characteristics}

Table 1 shows that both groups did not differ in all baseline characteristics, except for age and number of trajectories: Patients undergoing asleep surgery were older and less microelectrode trajectories were used.

Due to the retrospective nature of this study, $8.3 \%$ of data on TW measurements were missing; therefore, data of 96 patients were finally included in the analysis.
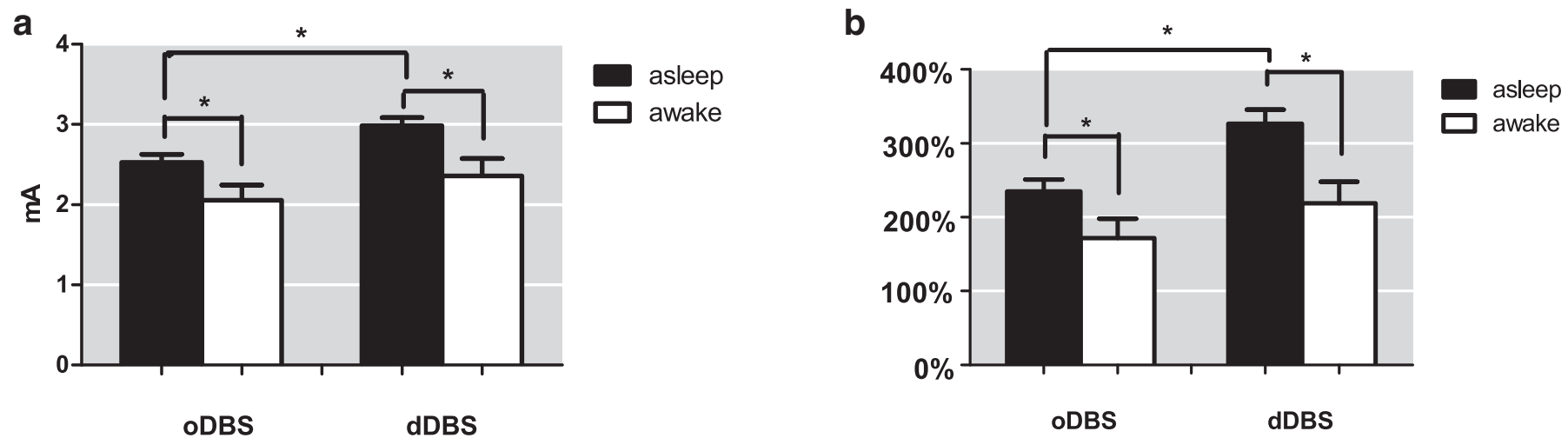

TW

TW $\%$

Figure 1 Therapeutic windows (mean \pm SE) (a) in absolute stimulation amplitude and (b) relative to therapeutic threshold, significance level was set to $p<0.025$. TW, therapeutic window; TW\%, therapeutic window percentage. 


\section{Therapeutic Window}

Absolute TW for the asleep group was significantly larger for both oDBS (asleep: $2.53 \pm 1.14 \mathrm{~mA}$, awake: $2.05 \pm 1.2 \mathrm{~mA}$, $p=0.009$ ) and dDBS (asleep: $2.99 \pm 1.05 \mathrm{~mA}$, awake: $2.36 \pm 1.28 \mathrm{~mA}, p=0.004)$ compared to the awake group. While in the awake group, TW was not different between dDBS and oDBS $(p=0.2)$, the asleep group showed a significantly wider TW with dDBS compared to oDBS ( $p=0.005$ ) (Fig. 1a).

TW\% for the asleep group was significantly wider for both oDBS (asleep: $234.7 \pm 185 \%$, awake $171.6 \pm 167 \%, p=0.015$ ) and
dDBS (asleep: $326.4 \pm 198 \%$, awake: $219 \% \pm 173 \%, p=0.0024$ ) compared to the awake group. While in the awake group TW\% was not different between oDBS and dDBS $(p=0.13)$, the asleep group showed a significantly wider TW\% with dDBS compared to oDBS ( $p=0.0001)$ (Fig. 1b).

\section{Therapeutic Threshold}

The mean $T$ did not differ between asleep and awake surgery for both oDBS (asleep $1.46 \pm 0.66 \mathrm{~mA}$, awake $1.65 \pm 0.80 \mathrm{~mA}$,

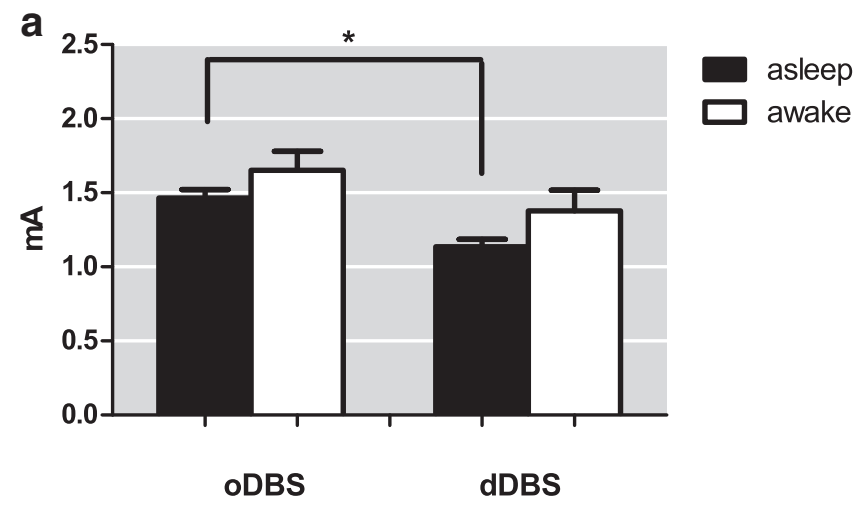

TT

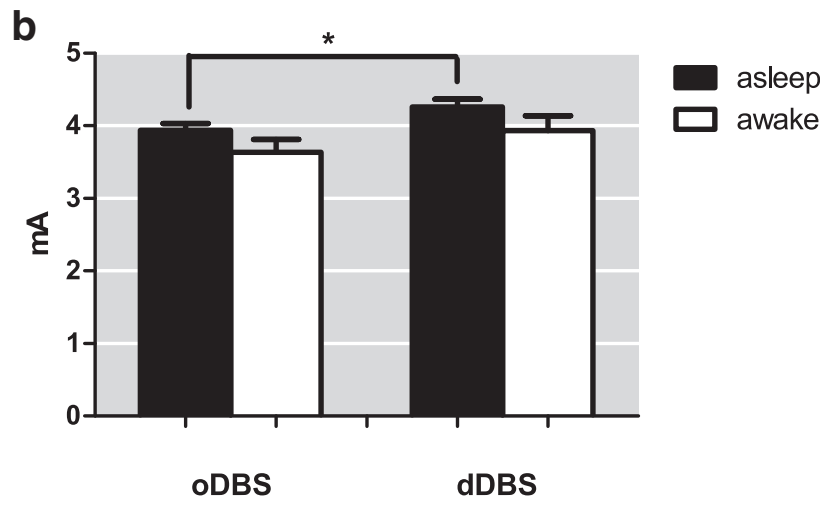

SET

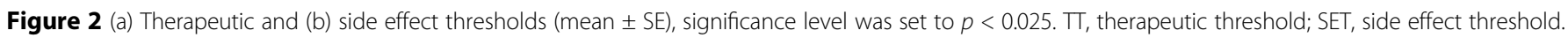

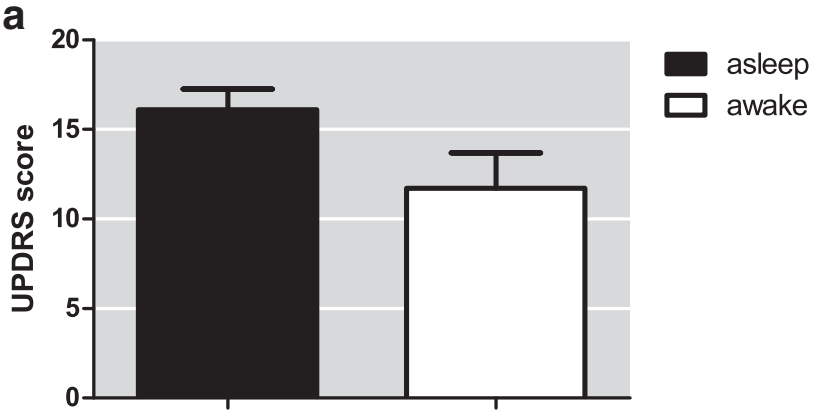

UPDRS improvement after 3 mo

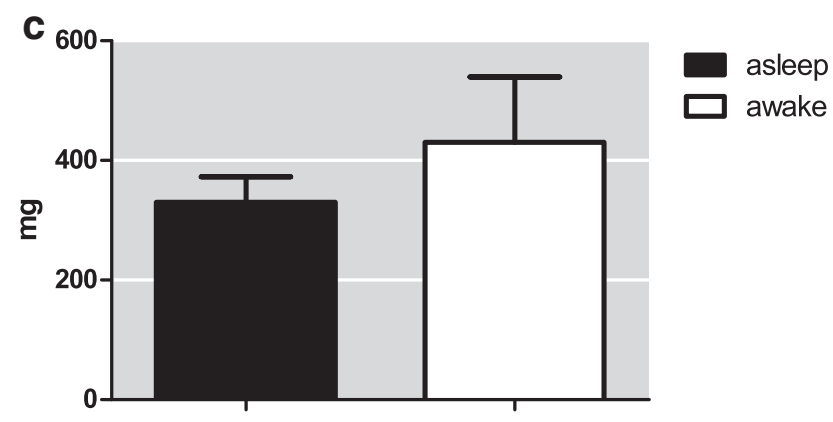

LEDD reduction after 3 mo

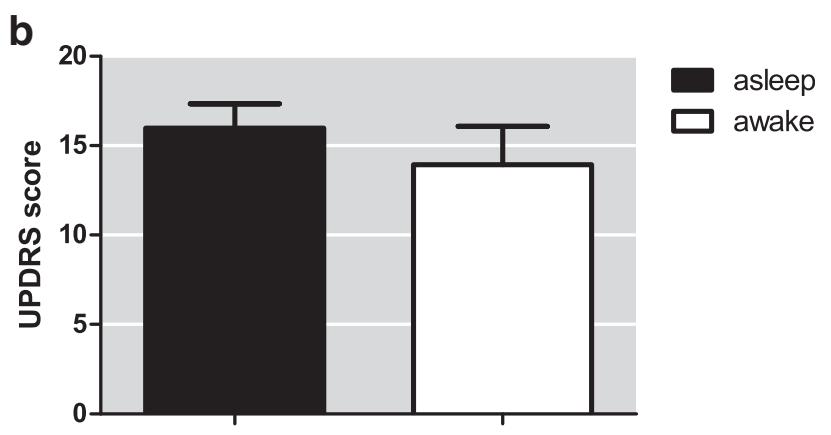

UPDRS improvement after 1 y

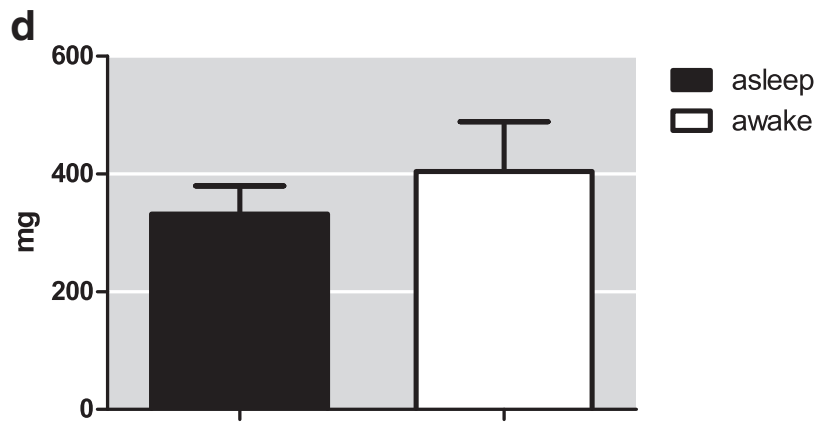

LEDD reduction after $1 \mathrm{y}$

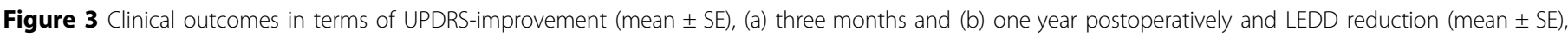

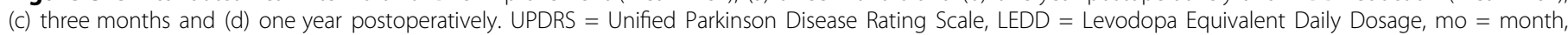
$y=$ year. 


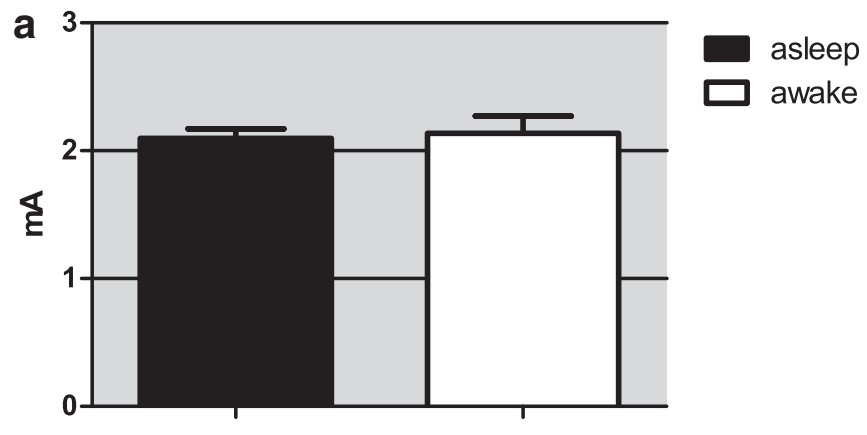

chronic stimulation amplitude 3 mo

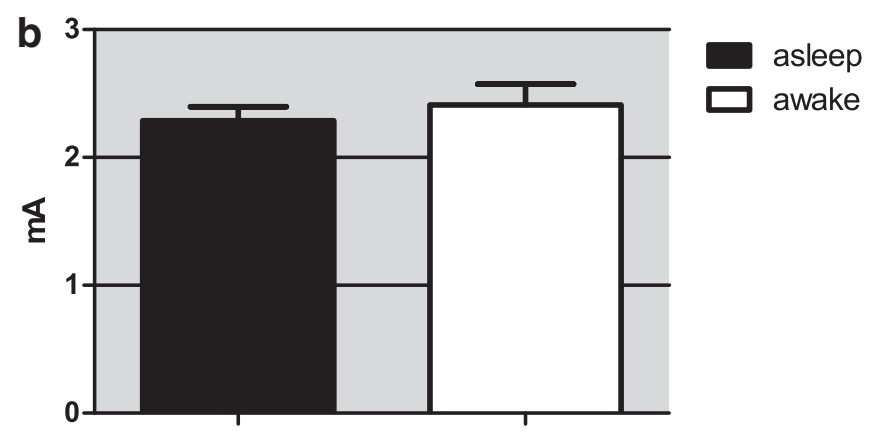

chronic stimulation amplitude 1 y

Figure 4 Chronic stimulation amplitude (mean \pm SE) (a) three months and (b) one year postoperatively. mo $=$ month, $y=y e a r$.

$p=0.29$ ) and dDBS (asleep $1.13 \pm 0.52 \mathrm{~mA}$, and awake $1.38 \pm 0.84 \mathrm{~mA}, p=0.14)$. In the asleep group, $\Pi$ was significantly lower for dDBS compared to oDBS $(p=0.0001)$, while in the awake group, no significant difference between oDBS and dDBS was found ( $p=0.09$ ) (Fig. 2a).

\section{Side Effect Threshold}

The mean SET did not differ between asleep and awake surgery for both oDBS (asleep: $3.94 \pm 1.09 \mathrm{~mA}$, awake: $3.64 \pm 1.16 \mathrm{~mA}$, $p=0.11$ ) and dDBS (asleep: $4.26 \pm 1.14 \mathrm{~mA}$, awake: $3.9 \pm 1.28 \mathrm{~mA}$, $p=0.16)$. In the asleep group, SET was significantly higher for dDBS compared to oDBS ( $p=0.016$ ), while in the awake group, no significant difference between dDBS and oDBS was found $(p=0.26)$ (Fig. 2b).

\section{Motor Improvement}

UPDRS III improvement after three months trended to be better in the asleep group but did not reach significance (asleep: $16.10 \pm 8.87$, awake: $11.71 \pm 8.12, p=0.06$ ). After one year (asleep: $15.97 \pm 7.96$, awake: $13.92 \pm 7.75, p=0.43)$ postoperatively there was no difference between groups (Fig. $3 a+b)$.

\section{Medication}

Preoperative LEDD showed no difference between both groups (asleep: $945 \pm 448 \mathrm{y}$, awake: $1027 \pm 689 \mathrm{y}, p=0.51$ ). The degree of postoperative LEDD reduction after both three months (asleep: $331 \pm 373 y$, awake: $430 \pm 514 y, p=0.31$ ) and one year (asleep: $332 \pm 349$ y, awake: $404 \pm 359$ y, $p=0.46$ ) did not differ between groups (Fig. $3 c+d$ ).

\section{Chronic Stimulation Amplitude}

Current intensity for chronic stimulation did not differ between asleep and awake group after three months (asleep: $2.10 \pm 0.9 \mathrm{~mA}$, awake: $2.14 \pm 0.85 \mathrm{~mA}, p=0.79$ ) as well as after one year (asleep: $2.29 \pm 1.01 \mathrm{~mA}$, awake: $2.41 \pm 0.89 \mathrm{~mA}, p=0.49$ ) (Fig. 4).

\section{DISCUSSION}

Our study has three main findings. First, asleep surgery was associated with wider therapeutic window. Second, in asleep surgery, dDBS allowed further increase of therapeutic window. Third, motor improvement, chronic stimulation amplitude and LEDD reduction within a year did not differ between asleep and awake surgery.

To the best of our knowledge, this is the first study investigating the influence of anesthesia type in terms of asleep and awake DBS surgery on therapeutic window $(21,22,26)$. Although increasing evidence including some meta analyses suggest noninferiority of asleep surgery regarding motor outcome, our results, however, support the notion of superiority of asleep surgery due to increase of TW (16-18). At first sight, this result may be surprising since awake surgery allows for an intraoperative testing of both $\Pi$ and SET, which should lead to optimal TW postoperatively. However, several aspects need to be taken into consideration explaining the difference between both groups.

First of all, asleep DBS may lead to higher accuracy with regard to the preoperative image-based targeting $(18,27)$. It is supposed that higher precision in asleep surgery may be achieved by reduction of brain shift (18). It is also important to mention that, contrast to other centers preferring asleep surgery based on anatomical targeting only, we also use MER in asleep surgery after reduction of anesthesia similar to the controlled general anesthesia technique described earlier (15). It is possible that in our patients MER and clinical testing for capsular side effects were already sufficient to determine the optimal target for implantation and further clinical testing had no additional impact. Moreover, given the fact that TW was smaller in the awake group, clinical testing could have even had a negative impact. One has to take into account, that adequate clinical evaluation of slight changes of muscle tone, oculomotor function or speech like the differentiation between PD associated dysarthria and DBS-induced dysarthria in the operation room setting with the patient off medication for more than 12 hours can be challenging even for an experienced neurologist specialized for clinical monitoring of DBS surgery (28). Finally, the mere fact that the patient undergoes clinical testing in the awake state can already lead to placebo effects leading to better intraoperative clinical outcome that may not be confirmed postoperatively $(29,30)$.

Our findings could also show that only in the asleep group dDBS can further increase TW by decreasing $T$ and increasing SET. Given the fact that for the awake group, TW also seemed to be wider for dDBS but without reaching statistical significance, we cannot rule out that our analysis was underpowered due to smaller sample size in the awake group. However, these results are generally consistent with previous studies which showed a superiority of directional compared to omnidirectional stimulation 
regarding therapeutic window $(7,11,26)$. Earlier reports have already shown $\mathrm{dDBS}$ to increase TW by either decreasing $\pi$ or increasing SET, but our study is the first to show both decrease of $\Pi$ and increase of SET at the same time with the same single segment. These results are consistent with a steeper increase of volume of neural activation with dDBS due to higher charge density of segmented contacts leading to lower $\Pi$ and at the same time support the notion of persistent directionality at higher stimulation intensities leading to higher SET $(7,9,26)$. Therefore, our results took the available findings one step further suggesting that directional DBS and asleep surgery may complement each other leading to the best TW.

Regarding clinical efficacy our results are consistent with earlier reports and recent meta analyses showing no relevant differences for motor outcome, reduction of medication and stimulation parameters between asleep and awake surgery (16,18-20,31-33). Wang et al. found no significant differences in clinical outcomes in terms of stimulation amplitude postoperatively and LEDD reduction or complications between the two techniques and concluded comparable surgical and clinical results of asleep to those of awake dDBS (18). Blasberg et al. could also show comparable results regarding stimulation amplitudes postoperatively and LEDD reduction between both groups (16). Ho et al. also did not find any difference in motor outcomes and LEDD (20). Nakajima et al. focused on percentage of improvement in UPDRS III motor scores, which did not show a difference between both groups as well (31).

The limitation of our study is its retrospective character. On the other hand, these results reflect real world clinical data. Another important point is that two characteristics differed at baseline. First, asleep patients were significantly older than awake patients. However, earlier studies have shown that older patients benefit less from DBS. In our study the older patients in the asleep group had even wider TW and therefore benefited more than the younger ones, suggesting that the age difference does not account for the results found here (34). Second, the number of trajectories in the asleep group was significantly lower than in the awake group. Since patients with advanced age or certain general medical conditions such as cardiovascular or respiratory problems are more likely to undergo asleep surgery, those might have had a higher chance of having hypertension or higher degree of brain atrophy and microangiopathy, which pose risk factors for surgery related hemorrhage. This may have resulted in the clinical decision to reduce the number of trajectories in order to minimize the risks for hemorrhages. On the one hand, we cannot fully rule out that surgery with less MER trajectories was less traumatizing in the asleep group and thus led to better outcome. Moreover, it is possible that an increased number of trajectories can somehow affect targeting accuracy. On the other hand, a reduced number of MER trajectories can also limit the ability to employ electrophysiological targeting. In this regard, Reck et al. could show an improvement of outcome using MER in DBS surgery (35). Since MER was used for both awake and asleep surgery, we cannot determine whether MER is helpful or harmful. However, this was actually not within the scope of this study and remains to be addressed further.

The focus of our study was the therapeutic window, which takes into account stimulation induced side effects and thus may be a more suitable outcome measure compared to a pure motor scoring. However, the advantages of wider TW for real life programming still needs to be confirmed in further long-term studies.

\section{CONCLUSION}

In conclusion, our study provides first evidence for enlarged TW by using asleep technique and dDBS, due to decreased $\Pi$ and increased SET, indicating an advantage compared to awake surgery. However, these results need to be confirmed further in prospective studies.

\section{Authorship Statements}

Stefan Jun Groiss and Farhad Senemmar designed the study which was conducted by Farhad Senemmar, Jan Vesper, Christian J. Hartmann, Philipp J. Slotty, Alfons Schnitzler, and Stefan Jun Groiss including patient recruitment, data collection, data analysis, and interpretation of data. Farhad Senemmar prepared the manuscript draft, Jan Vesper, Christian J. Hartmann, Philipp J. Slotty, Alfons Schnitzler, and Stefan Jun Groiss gave important intellectual input and critically revised the article. All authors approved the final manuscript.

\section{How to Cite this Article:}

Senemmar F., Hartmann C.J., Slotty P.J., Vesper J., Schnitzler A., Groiss S.J. 2021. Asleep Surgery May Improve the Therapeutic Window for Deep Brain Stimulation of the Subthalamic Nucleus.

Neuromodulation 2021; 24: 279-285

\section{REFERENCES}

1. von Campenhausen S, Bornschein B, Wick R et al. Prevalence and incidence of Parkinson's disease in Europe. Eur Neuropsychopharmacol 2005;15:473-490.

2. Balestrino R, Schapira AHV. Parkinson disease. Eur J Neurol 2020;27:27-42.

3. Lang AE, Lozano AM. Parkinson's disease. Second of two parts. N Engl J Med 1998;339:1130-1143.

4. Deep-Brain Stimulation for Parkinson's Disease Study Group, Obeso JA, Olanow CW et al. Deep-brain stimulation of the subthalamic nucleus or the pars interna of the globus pallidus in Parkinson's disease. N Engl J Med 2001;345: 956-963.

5. Geraedts VJ, van Hilten JJ, Marinus J et al. Stimulation challenge test after STN DBS improves satisfaction in Parkinson's disease patients. Parkinsonism Relat Disord 2019;69:30-33.

6. Dembek TA, Reker P, Visser-Vandewalle V et al. Directional DBS increases sideeffect thresholds-a prospective, double-blind trial. Mov Disord 2017;32:1380 1388.

7. Falconer R, Shah T, Rogers S, Green A, Shenai M. Utilizing the flexibility of directional deep brain stimulation intraoperatively (if needed) to minimize microelectrode lead repositioning. Cureus 2019;11:e5276.

8. Rebelo $P$, Green AL, Aziz TZ et al. Thalamic directional deep brain stimulation for tremor: spend less, get more. Brain Stimul 2018;11:600-606.

9. Bari AA, Fasano A, Munhoz RP, Lozano AM. Improving outcomes of subthalamic nucleus deep brain stimulation in Parkinson's disease. Expert Rev Neurother 2015; 15:1151-1160.

10. Shao MM, Liss A, Park YL et al. Early experience with new generation deep brain stimulation leads in Parkinson's disease and essential tremor patients. Neuromodulation 2019;23:537-542.

11. Bruno S, Nikolov P, Hartmann CJ, et al. Directional deep brain stimulation of the thalamic ventral intermediate area for essential tremor increases therapeutic window. Neuromodulation 2020. In press.

12. Hartmann CJ, Fliegen S, Groiss SJ, Wojtecki L, Schnitzler A. An update on best practice of deep brain stimulation in Parkinson's disease. Ther Adv Neurol Disord 2019;12:1756286419838096.

13. Schlaier JR, Habermeyer $C$, Janzen $A$ et al. The influence of intraoperative microelectrode recordings and clinical testing on the location of final stimulation sites in deep brain stimulation for Parkinson's disease. Acta Neurochir 2013;155:357-366.

14. Chen T, Mirzadeh Z, Ponce FA. "asleep" deep brain stimulation surgery: a critical review of the literature. World Neurosurg 2017;105:191-198. 
15. Fluchere F, Witjas T, Eusebio A et al. Controlled general anaesthesia for subthalamic nucleus stimulation in Parkinson's disease. J Neurol Neurosurg Psychiatry 2014;85:1167-1173.

16. Blasberg F, Wojtecki L, Elben $\mathrm{S}$ et al. Comparison of awake vs. asleep surgery for subthalamic deep brain stimulation in Parkinson's disease. Neuromodulation 2018;21:541-547.

17. Maltete $D$, Navarro $S$, Welter $M L$ et al. Subthalamic stimulation in Parkinson disease: with or without anesthesia? Arch Neurol 2004;61:390-392.

18. Wang J, Ponce FA, Tao J et al. Comparison of awake and asleep deep brain stimulation for Parkinson's disease: a detailed analysis through literature review. Neuromodulation 2020;23:444-450.

19. Liu Z, He S, Li L. General anesthesia versus local anesthesia for deep brain stimulation in Parkinson's disease: a meta-analysis. Stereotact Funct Neurosurg 2019;97 :381-390.

20. Ho AL, Ali R, Connolly ID et al. Awake versus asleep deep brain stimulation for Parkinson's disease: a critical comparison and meta-analysis. J Neurol Neurosurg Psychiatr 2018;89:687-691.

21. Moldovan AS, Hartmann CJ, Trenado $C$ et al. Less is more - pulse width dependent therapeutic window in deep brain stimulation for essential tremor. Brain Stimul 2018;11:1132-1139.

22. Steigerwald F, Muller L, Johannes S, Matthies C, Volkmann J. Directional deep brain stimulation of the subthalamic nucleus: a pilot study using a novel neurostimulation device. Mov Disord 2016;31:1240-1243.

23. Slotty PJ, Wille C, Kinfe TM, Vesper J. Continuous perioperative apomorphine in deep brain stimulation surgery for Parkinson's disease. Br J Neurosurg 2014;28: 378-382.

24. Tomlinson CL, Stowe R, Patel S, Rick C, Gray R, Clarke CE. Systematic review of levodopa dose equivalency reporting in Parkinson's disease. Mov Disord 2010;25: 2649-2653.

25. Schade S, Mollenhauer B, Trenkwalder C. Levodopa equivalent dose conversion factors - an updated proposal including opicapone and safinamide: LED for opicapone and safinamide. Mov Disord Clin Pract 2020;7:343-345.

26. Pollo C, Kaelin-Lang A, Oertel MF et al. Directional deep brain stimulation: an intraoperative double-blind pilot study. Brain 2014;137:2015-2026.

27. Mirzadeh Z, Chen T, Chapple KM et al. Procedural variables influencing stereotactic accuracy and efficiency in deep brain stimulation surgery. Oper Neurosurg (Hagerstown) 2019;17:70-78.

28. Holewijn RA, Verbaan D, de Bie RMA, Schuurman PR. General anesthesia versus local anesthesia in StereotaXY (GALAXY) for Parkinson's disease: study protocol for a randomized controlled trial. Trials 2017;18:417.

29. Walker HC, Faulk J, Rahman AF et al. Awake testing during deep brain stimulation surgery predicts postoperative stimulation side effect thresholds. Brain Sci 2019;9:44

30. Blume J, Schlaier J, Rothenfusser E et al. Intraoperative clinical testing overestimates the therapeutic window of the permanent DBS electrode in the subthalamic nucleus. Acta Neurochir 2017;159:1721-1726.

31. Nakajima T, Zrinzo L, Foltynie T et al. MRI-guided subthalamic nucleus deep brain stimulation without microelectrode recording: can we dispense with surgery under local anaesthesia? Stereotact Funct Neurosurg 2011;89:318-325.
32. Saleh S, Swanson KI, Lake WB, Sillay KA. Awake neurophysiologically guided versus asleep MRI-guided STN DBS for Parkinson disease: a comparison of outcomes using levodopa equivalents. Stereotact Funct Neurosurg 2015;93:419-426.

33. Brodsky MA, Anderson S, Murchison C et al. Clinical outcomes of asleep vs awake deep brain stimulation for Parkinson disease. Neurology 2017;89:1944-1950.

34. Dafsari HS, Reker P, Stalinski L et al. Quality of life outcome after subthalamic stimulation in Parkinson's disease depends on age. Mov Disord 2018;33:99-107.

35. Reck C, Maarouf M, Wojtecki L et al. Clinical outcome of subthalamic stimulation in Parkinson's disease is improved by intraoperative multiple trajectories microelectrode recording. J Neurol Surg A Cent Eur Neurosurg 2012;73:377-386.

\section{COMMENT}

The authors present a retrospective review of STN DBS patients undergoing directional DBS lead placement either awake or "asleep", i.e. under general anesthesia, and report that the therapeutic window was greater in those who underwent surgery via the "asleep" method. As the use of so-called "asleep DBS" increases, it is worthwhile noting that what these authors define as "asleep DBS" is by no means universally accepted, in that microelectrode recording (MER) was performed in both cohorts. Additionally, patients in the asleep group were taken off dopaminergic medications, the standard practice in awake DBS. Thus, two of the frequently touted advantages of asleep DBS, i.e. reduction in surgical risk by eliminating MER, and patient comfort by remaining on dopaminergic medications, are not applicable in this cohort. As in all retrospective reviews, concerns for selection bias exist - specifically, why were there fewer MER trajectories done in the asleep group? Could this have affected targeting accuracy? Nonetheless, it is worthwhile for the reader to gain insight into an experienced group's modification of time-honored surgical technique, all done to improve patient outcome.

Alon Y. Mogilner, MD, PhD New York, NY USA 